Canad. Math. Bull. Vol. 22 (4), 1979

\title{
ON THE INEQUALITY
}

$$
\begin{gathered}
\sum_{i=1}^{n} p_{i} \frac{f_{i}\left(p_{i}\right)}{f_{i}\left(q_{i}\right)} \leq 1 \\
\text { PETER KARDOS }
\end{gathered}
$$

1. Introduction. In this paper, we are concerned with the functional inequality

$$
\sum_{i=1}^{n} p_{i} \frac{f_{i}\left(p_{i}\right)}{f_{i}\left(q_{i}\right)} \leq 1
$$

where $0<p_{i}<1,0<q_{i}<1, f_{i}(p) \neq 0$, for $0<p<1,(i=1,2, \ldots, n) \sum_{i=1}^{n} p_{i}=$ $\sum_{i=1}^{n} q_{i}=1$, and $n$ is a fixed positive integer, $n \geq 2$.

Inequality (1) was studied by Rényi and Fischer, (see [1], [3]) in the special case

$$
\sum_{i=1}^{n} p_{i} \frac{f\left(p_{i}\right)}{f\left(q_{i}\right)} \leq 1
$$

and this provided a characterization of Rényi's entropy. Aczél considered a similar generalization of a similar but simpler and more fundamental inequality in [4].

Fischer has shown [1] that the general positive solution of (2) for $n \geq 3$ has the form

$$
f(p)=d p^{c} \quad \text { where } \quad d>0 \text { and }-1 \leq c \leq 0
$$

and he also investigated (2) for $f$ which may change signs. For $n=2$ in (2), Fischer proved that the general positive solution is monotone decreasing and continuous and in this case he also gave the general monotone decreasing solution with non-constant sign.

In this article, we give the general solution, with constant sign, to inequality (1) for fixed $n \geq 2$ and to inequality (2) when $n=2$. No regularity assumptions will be imposed on the functions.

2. The case $n \geq 3$. Our first theorem extends the results in [1] and [2].

TheOREM 1. Let $r \in(0,1]$ be fixed and let $f_{i}:(0,1) \rightarrow R, i=1,2$, satisfy the

Received by the editors June 19, 1978 and, in revised form, September 25, 1978. 
inequality

$$
p \frac{f_{1}(p)}{f_{1}(q)}+(r-p) \frac{f_{2}(r-p)}{f_{2}(r-q)} \leq r
$$

for all $p \in(0, r)$ and $q \in(0, r)$. If $f_{i}$ do not change signs, then each of the following hold:

(i) $f_{i}$ is monotonic decreasing (increasing) on $(0, r)$ if $f_{i}$ is positive (negative) on $(0,1)$.

(ii) $p \rightarrow p f_{i}(p)$ is increasing (decreasing) on $(0, r)$ if $f_{i}$ is positive (negative) on $(0,1)$.

(iii) $f_{i}$ is locally absolutely continuous on $(0, r)^{*}$.

(iv) if $f_{1}$ is differentiable at $p$ then $f_{2}$ is differentiable at $r-p$ and the following relation is valid:

$$
p \frac{f_{1}^{\prime}(p)}{f_{1}(p)}=(r-p) \frac{f_{2}^{\prime}(r-p}{f_{2}(e-p)} .
$$

Proof. We interchange $p$ and $q$ in (3) and obtain

$$
q \frac{f_{1}(q)}{f_{1}(p)}+(r-q) \frac{f_{2}(r-q)}{f_{2}(r-p)} \leq r .
$$

We can write (3) and (5) in the forms

$$
\frac{f_{2}(r-p)}{f_{2}(r-q)} \leq \frac{r-\left[p f_{1}(p) / f_{1}(q)\right]}{r-p} \text { and } \frac{f_{2}(r-q)}{f_{2}(r-p)} \leq \frac{r-\left[q f_{1}(q) / f_{1}(p)\right]}{r-q} .
$$

When we multiply these two inequalities, we get

$$
1 \leq \frac{\left[r f_{1}(q)-p f_{1}(p)\right]\left[r f_{1}(p)-q f_{1}(q)\right]}{(r-p)(r-q) f_{1}(p) f_{1}(q)}
$$

or, as $f_{1}$ does not change signs, that

Hence,

$$
r\left[f_{1}(q)-f_{1}(p)\right]\left[q f_{1}(q)-p f_{1}(p)\right] \leq 0 .
$$

$$
\left[f_{i}(q)-f_{i}(p)\right]\left[q f_{i}(q)-p f_{i}(p)\right] \leq 0
$$

for $i=1$, and by symmetry, for $i=2$. We shall now show (i), (ii), and (iii) in the case when $f_{i}$ is positive. If $f_{i}(p)<f_{i}(q)$ for some $p<q<r$ then the left-hand side of (6) would be positive. The contradiction implies that $f_{i}$ is decreasing on $(0, r)$. Moreover, if $p<q$ then $f_{i}(p) \geq f_{i}(q)$ and hence, by $(6), p f_{i}(p) \leq q f_{i}(q)$.

We prove that $f_{i}$ is locally absolutely continuous on $(0, r)$ in the case when $f_{i}$

\footnotetext{
* We wish to express our thanks to Prof. W. Walter for the simplification of the proof of (iii).
} 
is positive, $i=1,2$. Let $a, b, \varepsilon$ be fixed, $0<\varepsilon<a<b<r$, and let $s, t \in[a, b]$ be any two numbers, $s<t$. It follows from the monotonicity of $p \rightarrow p f_{i}(p)$ and $f_{i}$ that

Hence

$$
\begin{aligned}
0 \leq t f_{i}(t)-s f_{i}(s) & =(t-s) f_{i}(t)+s\left[f_{i}(t)-f_{i}(s)\right] \\
& \leq(t-s) f_{i}(t) \\
& \leq(t-s) f_{i}(\varepsilon) .
\end{aligned}
$$

$$
\left|t f_{i}(t)-s f_{i}(s)\right| \leq|t-s| f_{i}(\varepsilon) \text { for all } a \leq s<t \leq b
$$

and, by symmetry, (7) also holds for all $t<s$. Thus, $p \rightarrow p f_{i}(p)$ is Lipschitz on $[a, b]$ and therefore $f_{i}$ is locally absolutely continuous on $(0, r)$.

We prove (iv) in the case when $f_{2}>0$ on $(0,1)$. We may write (3) and (5) as

and

$$
(r-p) \frac{f_{2}(r-p)-f_{2}(r-q)}{f_{2}(r-q)} \leq p \frac{f_{1}(q)-f_{1}(p)}{f_{1}(q)}
$$

$$
(r-q) \frac{f_{2}(r-q)-f_{2}(r-p)}{f_{2}(r-p)} \leq q \frac{f_{1}(p)-f_{1}(q)}{f_{1}(p)}
$$

respectively. We deduce from these inequalities, if $r>q>p$, that

$$
\begin{aligned}
\frac{f_{2}(r-p)}{f_{1}(p)} & \cdot \frac{q}{r-q} \frac{f_{1}(q)-f_{1}(p)}{q-p} \leq \frac{f_{2}(r-q)-f_{2}(r-p)}{(r-q)-(r-p)} \\
& \leq \frac{f_{2}(r-q)}{f_{1}(q)} \cdot \frac{p}{r-p} \frac{f_{1}(q)-f_{1}(p)}{q-p} .
\end{aligned}
$$

Now, (iv) can be derived by letting $q \rightarrow p^{+}$. The case $q<p<r, q \rightarrow p^{-}$leads similarly to the desired result.

We give the general solution to (1) when each $f_{i}$ has constant sign and $n \geq 3$ in

Theorem 2. If $f_{i}(i=1,2, \ldots, n)$ do not change signs, then the general solution to (1) for fixed $n \geq 3$ has the form

$$
f_{i}(p)=b_{i} p^{a}, \quad i=1,2, \ldots, n
$$

where $-1 \leq a \leq 0$ and $b_{i}>0(<0)$ if $f_{i}>0(<0)$.

Proof. Put $p_{i}=q_{i}(i=3,4, \ldots, n)$ into (1). With

$$
p_{1}+p_{2}=q_{1}+q_{2}=r, p_{1}=p, q_{1}=q, p_{2}=r-p, q_{2}=r-q,
$$

(1) goes over into (3). Inequality (3) holds for all $p \in(0, r), q \in(0, r)$, and each $r \in(0,1)$. But then Theorem 1 (iv) means the following. If $f_{1}$ were not differentiable at a point $p_{0}$, then $f_{2}$ would not be differentaible at any $r-p_{0}$ 
$\left(r \in\left(p_{0}, 1\right)\right)$, that is, on the interval $\left(0,1-p_{0}\right)$. Since $f_{2}$ is monotonic, $f_{2}$ is differentiable almost everywhere on $(0,1)$. Hence $f_{1}$ and, by symmetry, also $f_{2}$ are differentiable on $(0,1)$ and (4) implies that

$$
p \frac{f_{1}^{\prime}(p)}{f_{1}(p)}=p \frac{f_{2}^{\prime}(p)}{f_{2}(p)}=a \quad \text { for all } \quad p \in(0,1)
$$

By solving for $f_{i}$ we obtain (8), $i=1,2$. Similarly, we can pair $f_{1}$ in turn with $f_{i}$, $i=3,4, \ldots, n$ and find that all $f_{i}$ are given by (8). Also, by Hölder's inequality,

$$
\sum_{i=1}^{n} p_{i} \frac{p_{i}^{a}}{q_{i}^{a}}=\sum_{i=1}^{n} p_{i}^{a+1} q_{i}^{-a} \leq\left(\sum_{i=1}^{n} p_{i}\right)^{a+1}\left(\sum_{i=1}^{n} q_{i}\right)^{-a}=1
$$

for $-1 \leq a \leq 0$ and in fact the opposite inequality holds when $a<-1$ or $a>0$.

3. The case $n=2$. In this section we give the general solution to (1) and (2) for $n=2$, when the functions do not change signs. For $n=2$, inequality (1) goes over into (3) with $r=1$.

Theorem 3. All solutions $f_{i}$ that do not change signs on $(0,1), i=1,2$, of the inequality

$$
p \frac{f_{1}(p)}{f_{1}(q)}+(1-p) \frac{f_{2}(1-p)}{f_{2}(1-q)} \leq 1, \quad 0<p<1,0<q<1
$$

are of the form

$$
f_{1}(p)=a \exp \left(\int_{c}^{p} \frac{(1-t) g^{\prime}(1-t)}{\operatorname{tg}(1-t)} d t\right), \quad f_{2}(p)=b g(p), p \in(0,1)
$$

where $a, b$, and $c$ are arbitrary, $a b \neq 0, c \in(0,1)$, with $g$ arbitrary continuous, positive, decreasing, and $p \rightarrow p g(p)$ increasing on $(0,1)$.

Proof. Let $f_{i}$ be solutions to (9) that do not change signs, say $f_{i}>0, i=1,2$. By Theorem $1, f_{2}$ is decreasing and continuous while $p f_{2}(p)$ is increasing on $(0,1)$. It follows from Theorem 1 (iii) and (iv) that

$$
p \frac{f_{1}^{\prime}(p)}{f_{1}(p)}=(1-p) \frac{f_{2}^{\prime}(1-p)}{f_{2}(1-p)}
$$

almost everywhere on $(0,1)$. Since $f_{2}$ is locally absolutely continuous on $(0,1)$, therefore $((1-t) / t)\left(f_{2}^{\prime}(1-t) / f_{2}(1-t)\right)$ is locally integrable on $(0,1)$ and we solve for $f_{1}$ in (11) to obtain (10) with $a=b=1$, and $f_{2}=g$. To prove the converse, it is enough to demonstrate that (10) satisfies (9) when $a=b=1$. Let $g$ be an arbitrary continuous, positive, decreasing function such that $\operatorname{pg}(p)$ is increasing. The argument in Theorem 1 (iii) shows that $g$ is locally absolutely continuous on $(0,1)$. We can prove that $p f_{1}(p)$ as defined in $(10)$ is increasing. Indeed, for 
$c>0$,

$$
\begin{aligned}
p f_{1}(p) & =p \exp \left(\int_{c}^{p} \frac{(1-t) g^{\prime}(1-t)}{\operatorname{tg}(1-t)} d t\right) \\
& =c \exp \left(\int_{c}^{p} \frac{(1-t) g^{\prime}(1-t)}{\operatorname{tg}(1-t)} d t+\int_{c}^{p} \frac{1}{t} d t\right) \\
& =c \exp \left(\int_{c}^{p} \frac{(1-t) g^{\prime}(1-t)+g(1-t)}{\operatorname{tg}(1-t)} d t\right) .
\end{aligned}
$$

As $p g(p)$ is increasing, $d[p g(p)] / d p=p g^{\prime}(p)+g(p) \geq 0$ almost everywhere and, as $g$ is positive,

$$
\int_{p_{1}}^{p_{2}} \frac{(1-t) g^{\prime}(1-t)+g(1-t)}{\operatorname{tg}(1-t)} \geq 0
$$

for all $0<p_{1}<p_{2}<1$. From (12) and (13) we have

$$
p_{1} f_{1}\left(p_{1}\right) \leq p_{2} f_{1}\left(p_{2}\right) \text { for } 0<p_{1}<p_{2}<1
$$

and thus $p f_{1}(p)$ is increasing. By differentiating $f_{1}$ in (10), we obtain

$$
\frac{t f_{1}^{\prime}(t)}{f_{1}(t)}=(1-t) \frac{g^{\prime}(1-t)}{g(1-t)}
$$

a.e. on $(0,1)$, say for all $t \in A$. Define

$$
H(t)=\frac{t f_{1}^{\prime}(t)}{f_{1}(t)}=(1-t) \frac{g^{\prime}(1-t)}{g(1-t)}, t \in A ;
$$

then $H(t) \leq 0, t \in A$. Let $p, q$ be fixed, $1>q>p>0$. Because $p f_{1}(p)$ and $p g(p)$ are increasing, we find in logical sequence, for $t \in A \cap[p, q]$, that

$$
\begin{gathered}
\frac{p f_{1}(p)}{t f_{1}(t)} \leq 1 \leq \frac{(1-p) g(1-p)}{(1-t) g(1-t)}, \\
H(t) \frac{p f_{1}(p)}{t f_{1}(t)} \geq H(t) \frac{(1-p) g(1-p)}{(1-t) g(1-t)}, \\
p \frac{f_{1}(p) f_{1}^{\prime}(t)}{f_{1}(t)^{2}} \geq(1-p) \frac{g(1-p) g^{\prime}(1-t)}{g(1-t)^{2}}, \\
p f_{1}(p) \int_{p}^{a} \frac{f_{1}^{\prime}(t)}{f_{1}(t)^{2}} d t \geq(1-p) g(1-p) \int_{p}^{a} \frac{g^{\prime}(1-t)}{g(1-t)^{2}} d t, \\
p f_{1}(p)\left[-\frac{1}{f_{1}(t)}\right]_{p}^{a} \geq(1-p) g(1-p)\left[-\frac{1}{g(t)}\right]_{1-q}^{1-p},
\end{gathered}
$$

and that

$$
1 \geq p \frac{f_{1}(p)}{f_{1}(q)}+(1-p) \frac{g(1-p)}{g(1-q)}=p \frac{f_{1}(p)}{f_{1}(q)}+(1-p) \frac{f_{2}(1-p)}{f_{2}(1-q)},
$$

which is (9). Similarly, (9) holds if $q<p$. 
We now give the general solution to inequality (2), when $n=2$, if $f$ has constant sign.

THEOREM 4. All solutions that do not change signs on $(0,1)$, of the inequality

$$
p \frac{f(p)}{f(q)}+(1-p) \frac{f(1-p)}{f(1-q)} \leq 1, \quad 0<p<1, \quad 0<q<1,
$$

are of the form

$$
f(p)=a \exp \left(\int_{b}^{p} \frac{G(t)}{t} d t\right), \quad p \in(0,1),
$$

where $a \neq 0, b \in(0,1)$ with $G$ arbitrary measurable on $(0,1)$ and satisfying for almost all $p \in(0,1)$

$$
G(1-p)=G(p)
$$

and

$$
-1 \leq G(p) \leq 0 \text {. }
$$

Proof. We may suppose that $f>0$ on $(0,1)$. We shall use Theorem 1 with $f=f_{1}=f_{2}$ and $r=1$. It follows from (i) that $f$ is differentiable a.e. on $(0,1)$. Then, by (iv) we have that

$$
p \frac{f^{\prime}(p)}{f(p)}=(1-p) \frac{f^{\prime}(1-p)}{f(1-p)}
$$

for almost all $p$ on $(0,1)$, say on $A$. Define

$$
G(p)=p \frac{f^{\prime}(p)}{f(p)}, \quad p \in A ;
$$

then (16) holds. Moreover, by (ii), $p f(p)$ is increasing so that

Thus

$$
f(p)+p f^{\prime}(p) \geq 0, \text { for } p \in A \text {. }
$$

$$
1+G(p) \geq 0, \quad p \in A,
$$

and since $f^{\prime}(p) \leq 0,(17)$ is valid. We obtain from (iii) that $f$ is locally absolutely continuous on $(0,1)$. Therefore $G$ is measurable and

$$
\frac{G(p)}{p}=\frac{f^{\prime}(p)}{f(p)}
$$

is locally integrable on $(0,1)$. We derive (15) by integrating (18). It can be shown, as in Theorem 3 , that all $f$ given by (15) satisfy (14).

\section{REFERENCES}

1. P. Fischer, On the inequality $\sum_{i=1}^{n} p_{i}\left(f\left(p_{i}\right) / f\left(q_{i}\right)\right) \leq 1$, Canadian Math. Bull. 17 (1974), 193199. 
2. J. Aczél and Z. Daróczy, On Measures of Information and their Characterizations, Academic Press, New York, 1975.

3. A. Rényi, On the Foundations of Information Theory, Rev. Inst. Internat. Stat. 33 (1965), $1-14$.

4. J. Aczél, General solution of an inequality containing several unknown functions, with applications to the generalized problem of how to keep the (inset) expert honest, Notices Amer. Math. Soc. vol. 25, nr. 4, p. A-435, \#78T-C18.

\section{Physical Sciences Group \\ Scarborough College \\ UNIVERSITY OF TORONTO \\ WEST HILl, ONTARIO \\ MIC 1A4}

